Geremy Carlos Freitas

Análise da Potencialidade de uso de um Solo Siltoso para Utilização em Barreira Mineral de Aterros Sanitários no Estado de Roraima

Dissertação apresentada como requisito parcial para obtenção do grau de Mestre pelo Programa de PósGraduação em Engenharia Civil do Departamento de Engenharia Civil da PUC-Rio.

Orientador: Prof. José Tavares Araruna Júnior 
Geremy Carlos Freitas

\section{Análise da Potencialidade de uso de um Solo Siltoso para Utilização em Barreira Mineral de Aterros Sanitários no Estado de Roraima}

Dissertação apresentada como requisito parcial para obtenção do grau de Mestre pelo Programa de Pós-Graduação em Engenharia Civil do Departamento de Engenharia Civil do Centro Técnico Científico da PUC-Rio. Aprovada pela Comissão Examinadora abaixo assinada.

Prof. José Tavares Araruna Junior Orientador Departamento de Engenharia Civil - PUC-Rio

Prof. Alberto de Sampaio Ferraz Jardim Sayão Departamento de Engenharia Civil - PUC-Rio

Prof. Gustavo Ferreira Simões Universidade Federal de Minas Gerais

Prof. José Eugenio Leal Coordenador Setorial do Centro Técnico Científico da PUC-Rio

Rio de Janeiro, 04 de agosto de 2015. 
Todos os direitos reservados. É proibida a reprodução total ou parcial do trabalho sem a autorização da universidade, da autora e do orientador.

\section{Geremy Carlos Freitas}

Gradou-se em Engenharia Ambiental e Sanitária na Pontifícia Universidade Católica do Rio de Janeiro, com período sanduíche no Institut National des Sciences Appliquées de Lyon, França, no período de 2011/2012, onde cursou disciplinas sobre a gestão e os diversos tipos de tratamento de resíduos sólidos. A experiência no exterior aliada ao conhecimento adquirido durante a graduação permitiu realizar partes do Plano Municipal de Gestão Integrada de Resíduos Sólidos de uma cidade localizada no norte do Brasil, como Trabalho de Conclusão de Curso e motivou a pósgraduação na área de geotecnia ambiental.

\section{Ficha Catalográfica}

\section{Freitas, Geremy Carlos}

Análise da potencialidade de uso de um solo siltoso para utilização em barreira mineral de aterros sanitários no estado de Roraima / Geremy Carlos Freitas ; orientador: José Tavares Araruna Junior. -2015.

202 f. il. (color.) ; $30 \mathrm{~cm}$

Dissertação (mestrado)-Pontifícia Universidade Católica do Rio de Janeiro, Departamento de Engenharia Civil, 2015.

Inclui bibliografia

1. Engenharia civil - Teses. 2. Aterro sanitário. 3. Barreira mineral. 4. Condutividade hidráulica. 5. Contração. 6. Resistência à compressão simples. 7. Resistência ao cisalhamento. 8. Solos de Roraima. I. Araruna Junior, José Tavares. II. Pontifícia Universidade Católica do Rio de Janeiro. Departamento de Engenharia Civil. III. Título. 


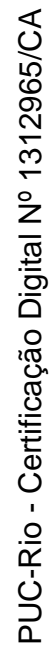

Ao meu querido Jesus Cristo, sem o qual eu nada posso fazer. 


\section{Agradecimentos}

Agradeço a Deus, o todo poderoso, pois sem Ele nada teria acontecido.

Aos meus pais, Sebastião e Arlete Freitas, pela vida, amor, educação, dedicação e carinho;

Aos meus irmãos, Geder e Gezer Freitas, as minhas cunhadas Jéssica Tavares e Jucy, pelo carinho e apoio e aos meus sobrinhos, Isabelly e Pedro pela sua simples existência em nossas vidas;

À minha grande família em Juiz de Fora, especialmente ao meu avô Sebastião Mendes, in memoriam, pelos ensinamentos valiosos ao longo de sua vida cristã exemplar;

À Primeira Igreja Batista de Rorainópolis pelas orações e incentivo;

Ao Irmão Ademar Maia e sua família pela alegria em cada chegada e a força em cada partida de Manaus;

À Igreja Batista em Botafogo, especialmente aos Pastores Márcio Mattos, Adauberto e Thiago Titillo e ao Vice-moderador Abner e a Juventude PIB Botafogo pelo acolhimento segundo os preceitos bíblicos;

À Salete Rodrigues e sua família pelo amor e cuidado demonstrado no dia a dia;

Aos meus grandes amigos Érica, Helen, Lucas, Maria e Rômulo pelo auxílio nas batalhas e momentos únicos de oração e comunhão verdadeira; 
Aos amigos Rodrigo, Elaine, Mariana, Marcelo Magalhães e tantos outros com os quais caminhei ao longo desses dois anos e meio;

Ao pessoal do Laboratório de Geotecnia da PUC: Edison, Amaury, Josué, Carlos, Victor, Elizânio e os demais.

Ao Professor Araruna, pela orientação, compreensão, paciência e amizade;

À PUC-Rio e ao CNPQ, pelos auxílios concedidos, sem os quais este trabalho não poderia ter sido realizado.

A todos que de forma direta ou indireta contribuíram para que mais essa vitória fosse conquistada. 


\section{Resumo}

Freitas, Geremy Carlos; Araruna Júnior, José Tavares. Análise da Potencialidade de uso de um Solo Siltoso para Utilização em Barreira Mineral de Aterros Sanitários no Estado de Roraima. Rio de Janeiro, 2015. 202p. Dissertação de Mestrado - Departamento de Engenharia Civil, Pontifícia Universidade Católica do Rio de Janeiro.

A Lei da Política Nacional de Resíduos Sólidos exigiu a extinção dos lixões e aterros controlados nos municípios brasileiros. Embora a lei date de 2010, até agora a maioria dos municípios brasileiros ainda não conseguiu implantar aterros sanitários. A situação não é diferente no Estado de Roraima, onde nenhum de seus 15 (quinze) municípios possui um aterro sanitário em operação. Este trabalho visa a minimizar esta ocorrência, à medida que estuda as propriedades e índices geotécnicos de um solo siltoso de uma jazida do município de Rorainópolis que poderá ser empregado como material de construção de barreiras minerais de um possível Aterro Consorciado que atenderá os municípios que compõem a Região Metropolitana do Sul de Roraima, a saber: Rorainópolis, São João da Baliza, São Luís e Caroebe. O solo siltoso foi submetido a um extenso programa experimental que consistiu de ensaios de condutividade hidráulica, compressão simples e contração em corpos de prova, com diferentes relações massa-volume-umidade, compactados nas energias Proctor normal, intermediária e modificada. O programa experimental objetivou estabelecer uma faixa de variação de valores da relação massa-volume-umidade que atendesse aos critérios usualmente empregados na especificação de construção de barreiras minerais, i.e. condutividade hidráulica igual ou inferior a $10^{-9} \mathrm{~m} / \mathrm{s}$, resistência à compressão simples superior a $200 \mathrm{KPa}$ e contração volumétrica inferior a 4\%. Os resultados do programa experimental demostraram que o solo deverá possuir um peso específico de $14,75 \pm 0,5 \mathrm{KN} / \mathrm{m}^{3}$ e um teor de umidade de $28,0 \pm 3,0 \%$, a fim de atender aos critérios de condutividade hidráulica e resistência. Os resultados mostraram que não é possível atender ao critério de contração volumétrica, face à presença de argilominerais 2:1 na composição do solo estudado. Não obstante, é 
possível empregá-lo com essa finalidade, desde que cuidados sejam adotados para evitar a sua dessecação. Adicionalmente, foram determinados os parâmetros de resistência ao cisalhamento do solo através de ensaios de cisalhamento direto a fim de possibilitar a análise de estabilidade de taludes.

\section{Palavras-chave}

Aterro sanitário; barreira mineral; condutividade hidráulica; contração; resistência à compressão simples; resistência ao cisalhamento; Solos de Roraima. 


\section{Abstract}

Freitas, Geremy Carlos; Araruna Júnior, José Tavares (Advisor). An Assessment of the potential use of a compacted silty soil from Roraima State as a landfill mineral barrier. Rio de Janeiro, 2015. 202p. MSc Dissertation - Departamento de Engenharia Civil, Pontifícia Universidade Católica do Rio de Janeiro.

The Law of the National Solid Waste Policy demanded the extinction of controlled dumps and landfills in Brazilian municipalities. Although the date 2010 law, so far most municipalities have yet to deploy landfills. The situation is no different in the state of Roraima, where none of his fifteen (15) municipalities has a landfill in operation. This work aims to minimize this occurrence, as studying the properties and geotechnical contents of a clay soil deposit of Rorainópolis municipality that may be used as a building material mineral barriers of a possible Syndicated landfill that will serve the municipalities that make up the metropolitan area of southern Roraima, namely: Rorainópolis, São João da Baliza, São Luís and Caroebe. The clay soil was subjected to an comprehensive experimental program consisting of hydraulic conductivity tests, simple compression and contraction on specimens with different mass-volume-moisture relations, compacted in standard, intermediate and modified energies. The experimental program aimed to establishing a range of variation of volume-massmoisture values that met the criteria usually employed in the construction specification of mineral barriers, i.e. hydraulic conductivity equal to or less than $10^{-9} \mathrm{~m} / \mathrm{s}$, compressive resistance higher than $200 \mathrm{KPa}$ and volumetric shrinkage of less than $4,0 \%$. The results of the experimental program demonstrated that the soil must have a specific weight of $14.75 \pm 0.5 \mathrm{KN} / \mathrm{m}^{3}$, and a moisture of $28.0 \pm$ $3.0 \%$ content, in order to meet the criteria hydraulic conductivity and resistance. The results show that the volumetric shrinkage criterion cannot be meet due to the presence of 2: 1 clay soil composition studied. Nevertheless, it is possible to use it as a building material mineral barrier, as long as care is adopted to prevent 
desiccation. In addition it was determined the soil shear parameters of resistance through direct shear tests to enable the slope stability analysis.

\section{Keywords}

Landfill; impermeable barrier; hydraulic conductivity; contraction; resistance to simple compression; shear strength; Soils of Roraima. 


\section{Sumário}

1 Introdução 26

1.1. Organização da Dissertação 27

2 Revisão Bibliográfica 29

2.1. Resíduos Sólidos $\quad 29$

2.2. Disposição Final de Resíduos Sólidos 31

2.3. Aterros Sanitários 38

2.4. Sistemas de Impermeabilização de Base 46

2.4.1. Barreiras Minerais com Solo Natural 47

2.4.2. Barreiras Minerais com Geossintéticos $\quad 50$

2.4.3. Barreiras Minerais com Solo Compactado 50

2.4.3.1. Características dos Solos 51

2.5. Condutividade Hidráulica de Solos $\quad 60$

2.5.1. Fatores que Afetam a Condutividade Hidráulica de Solos 60

2.5.2. Equipamentos para a Obtenção da Condutividade Hidráulica de $\begin{array}{ll}\text { Solos } & 68\end{array}$

2.6. Contração dos Solos 72

2.7. Resistência ao Cisalhamento em Barreiras Minerais 76

$\begin{array}{ll}\text { 2.7.1. Fatores que Afetam a Resistência ao Cisalhamento } & 78\end{array}$

3 Materiais e métodos $\quad 79$

3.1. Materiais $\quad 79$

3.2. Métodos 87

3.2.1. Ensaios de Caracterização Física $\quad 87$

$\begin{array}{ll}\text { 3.2.2. Ensaios de Caracterização Química } & 88\end{array}$

3.2.3. Ensaios de Caracterização Mineralógica 91

3.2.4. Ensaios de Caracterização Mecânica 94

3.2.5. Ensaios de Caracterização Hidráulica 100

$\begin{array}{ll}\text { 3.2.6. Ensaios de Contração } & 104\end{array}$ 
4 Apresentação e análise dos resultados 107

4.1. Caracterização Geotécnica 107

4.2. Caracterização Química 110

4.3. Caracterização Mineralógica 113

4.4. Ensaios de Compactação 115

4.4.1. Ensaio de Compactação Com Reuso 115

4.4.2. Ensaio de Compactação sem Reuso 116

4.5. Ensaios de Condutividade Hidráulica 119

$\begin{array}{ll}\text { 4.6. Ensaios de Contração } & 137\end{array}$

4.7. Ensaios de Compressão Simples (ou Não Confinada) 146

4.8. Ensaios de Cisalhamento Direto 150

4.9. Definição da Área Admissível 159

5 Conclusões e Sugestões para Trabalhos Futuros 194

6 Referências Bibliográficas 165

Anexo A - Resultados das análises de microscopia eletrônica de varredura 171

Anexo B - Relação entre contração axial e a variação da temperatura e da 181 umidade relativa do ar

Anexo c - Resultados dos Ensaios de Cisalhamento direto 


\section{Lista de tabelas}

Tabela 1: Diferentes formas de Classificação dos Resíduos Sólidos, 30 IPT/CEMPRE (2000).

Tabela 2: Situação de municípios roraimenses quanto à disposição final de resíduos sólidos, dados obtidos a partir do Portal da Transparência do Governo Federal (transparencia.gov.br).

Tabela 3: Critérios para seleção de áreas para instalação de aterros sanitários, IPT (1995).

Tabela 4: Classificação dos aterros sanitários com base na Taxa máxima de deposição, Gariglio (2003).

Tabela 5: Critérios para a dispensa de impermeabilização complementar de acordo com a NBR 15849 (ABNT, 2010).

Tabela 6: Valores de Excedente Hídrico entre os anos 1961 e 2008, para o município de Rorainópolis Roraima.

Tabela 7: Diferenças entre as geomembranas de polietileno e de PVC (apud MACAMBIRA, 2002).

Tabela 8: CTC e Superfície Específica dos principais argilominerais e frações silte e argila modificado de Costa (2002).

Tabela 9: Condutividade hidráulica dos argilominerais (adaptado de MESRI e OLSON, 1971).

Tabela 10: Vantagens e desvantagens de Permeâmetros, modificado de Daniel (1994).

Tabela 11: Variação entre valores de condutividade hidráulica obtida no campo e em ensaios de laboratório (DANIEL, 1987).

Tabela 12: Classificação das trincas conforme sua magnitude (KLEPPE e OLSON, 1985).

Tabela 13: Programa Experimental.

Tabela 14: Resumo das características granulométricas dos materiais, em porcentagem.

Tabela 15: Limites de Consistência (\%), índice de plasticidade e 
atividade.

Tabela 16: Capacidade de Troca Catiônica e Superfície Específica. $\quad 110$

Tabela 17: Superfície específica de alguns argilominerais, LCPC (1979). 110

Tabela 18: CTC para alguns argilominerais, adaptado de Stevenson 110 (1985)

Tabela 19: Teor de Matéria Orgânica e pH.

Tabela 20: Concentrações dos principais óxidos e elementos do solo.

Tabela 21: Elementos presentes em alguns corpos de prova.

Tabela 22: Valores de Umidade Ótima e $\gamma d$ máx, para os ensaios com reuso.

Tabela 23: Valores de Umidade Ótima e $\gamma d$ máx, para os ensaios sem reuso.

Tabela 24: Resumo dos valores de Umidade Ótima e $\gamma \mathrm{d}$ máx, para os ensaios com e sem reuso.

Tabela 25: Condições iniciais, parâmetros de ensaio e condutividade hidráulica das amostras compactadas na Energia do Proctor Normal.

Tabela 26: Condições iniciais, parâmetros de ensaio e condutividade hidráulica das amostras compactadas na Energia do Proctor Intermediária.

Tabela 27: Condições iniciais, parâmetros de ensaio e condutividade hidráulica das amostras compactadas na Energia do Proctor Modificada.

Tabela 28: Dados dos ensaios de cisalhamento direto para a Energia do Proctor Normal.

Tabela 29: Dados dos ensaios de cisalhamento direto para a Energia do Proctor Intermediária.

Tabela 30: Dados dos ensaios de cisalhamento direto para a Energia do Proctor Modificada.

Tabela 31: Resultados dos ensaios de cisalhamento para as energias do Proctor normal, intermediária e modificada.

Tabela 32: Parâmetros dos pontos inseridos na zona admissível. 


\section{Lista de figuras}

Figura 1: Aumento da geração de resíduos sólidos entre os anos de 2012 e 31 2013 (ABRELP, 2013).

Figura 2: Geração de resíduos sólidos de acordo com a região (ABRELP, 2013).

Figura 3: Lixão ou vazadouro (FEAM, 2010).

Figura 4: Destinação final de resíduos sólidos no Brasil (ABRELP, 2013). 34

Figura 5: Destinação final de resíduos sólidos no Brasil (ABRELP, 2013). 34

Figura 6: Destinação final de resíduos sólidos na região Norte do Brasil 35 (ABRELP, 2013).

Figura 7: Destinação final de resíduos sólidos em Roraima (ABRELP, 36 2013).

Figura 8: Vista de um aterro sanitário, Manual de Operações de Aterros Sanitários (CONDER, 2002).

Figura 9: Aterro em trincheiras, Caderno técnico de reabilitação de áreas degradadas por resíduos sólidos urbanos (FEAM, 2010).

Figura 10: Espalhamento e compactação dos resíduos sólidos, Manual de Operações de Aterros Sanitários (CONDER, 2002).

Figura 11: Aterro em Rampa, Caderno técnico de reabilitação de áreas degradadas por resíduos sólidos urbanos (FEAM, 2010).

Figura 12: Aterro Sanitário, adaptado do site do Governo do Estado de Rondônia (www.rondonia.ro.gov.br).

Figura 13: Requisitos legais para resíduos não perigosos (ISSMGE, 2006).

Figura 14: Requisitos legais para resíduos perigosos (ISSMGE, 2006).

Figura 15: Método Tradicional para especificação da Zona Admissível mediante teor de umidade e peso específico seco (DANIEL e BENSON, 1999).

Figura 16: Área aceitável segundo a abordagem tradicional, transpassada 
por linhas de mesma condutividade hidráulica (DANIEL e BENSON, 1990).

Figura 17: Procedimentos para obtenção da área admissível (DANIEL e 54 BENSON, 1990).

Figura 18: Aplicação do método de definição da área admissível (BENSON e DANIEL, 1990).

Figura 19: Aplicação do método de definição da área admissível, acrescentando os parâmetros de resistência ao cisalhamento e contração volumétrica. (BENSON e DANIEL, 1990).

$\begin{array}{lllll}\text { Figura 20: Melhores formas de compactação das camadas } 57 & 57\end{array}$ impermeabilizantes de fundo de aterros sanitários (McBean et al., 1995).

Figura 21: Probabilidade de a condutividade hidráulica exceder 1,0 x 10-7 58 $\mathrm{cm} / \mathrm{s}$ em função da espessura da camada (BENSON e DANIEL, 1994).

Figura 22: Conectividade hidráulica.

Figura 23: Efeito da compactação na estrutura interna do solo, (LAMBE e WHITMAN, 1979).

Figura 24: Variação da condutividade hidráulica em função do teor de umidade (LAMBE e WHITMAN, 1979).

Figura 25: Resultados de condutividade hidráulica e massa específica seca e umidade de moldagem (MITCHELL et al., 1965).

Figura 26: Fluxo de água através de macroporos, entre os grumos (OLSEN, 1962).

Figura 27: Condutividade hidráulica para solos com diâmetros máximos de torrões de

Figura 28: Variação da condutividade hidráulica devido ao amento da energia de compactação (MITCHELL et al., 1965).

Figura 29: Relação entre condutividade hidráulica e fração granulométrica do solo (BENSON et al., 1994).

Figura 30: Relação entre condutividade hidráulica e índice de plasticidade (BENSON et al., 1994).

Figura 31: Relação entre condutividade hidráulica e atividade (BENSON et al., 1994).

Figura 32: Permeâmetro de parede rígida (DANIEL, 1994). 
Figura 33: Permeâmetro de parede flexível (DANIEL, 1994).

Figura 34: Relação entre trincas e contração volumétrica (KLEPPE e 73 OLSON, 1985).

Figura 35: Variação da contração volumétrica em função do teor de umidade de moldagem para diferentes energias de compactação (ALBRECHT e BENSON, 2001).

Figura 36: Principais configurações utilizadas em aterros sanitários (SHARMA e LEWIS, 1994).

Figura 37: Superfícies de ruptura que ocorrem em aterros sanitários (SHARMA e LEWIS, 1994).

Figura 38: Localização do Estado de Roraima e do município de

Rorainópolis. Fonte: Instituto Nacional de Pesquisas da Amazônia - INPA.

Figura 39: Localização do ponto de coleta da amostra. Fonte: Google Earth.

Figura 40: Foto do local de amostragem.

Figura 41: Perfil do solo da jazida.

Figura 42: Acondicionamento da amostra para o transporte.

Figura 43: Mapa de localização do município de Rorainópolis dentro do contexto do (a) Escudo das Guianas e dos (b) domínios tectonoestratigráficos de Roraima (apud ALMEIDA, 2006).

Figura 44: Domínios lito estruturais de Roraima (IBGE, 2005).

Figura 45: Encarte tectônico de parte de Roraima (adaptado de CPRM, 2004).

Figura 46: Recorte do mapa geológico do Estado de Roraima com destaque para a região de coleta da amostra e legenda de interesse (CPRM, 2004).

Figura 47: Agitador mecânico e materiais para o Ensaio de Azul de Metileno.

Figura 48: Execução e resultado do Ensaio de Azul de Metileno.

Figura 49: Equipamento de medição do pH (Fonte: 91 www.tecnopon.com.br).

Figura 50: Equipamento Hitachi TM - 1000, utilizado nas análises de MEV.

Figura 51: Preparação das lâminas pelo método do gotejamento. 
Figura 52: Obtenção de amostras para as análises de MEV. 93

$\begin{array}{ll}\text { Figura 53: Resultado de uma das análises de MEV. } & 94\end{array}$

Figura 54: Ensaio de compactação.

Figura 55: Prensa de Cisalhamento Direto pertencente ao Laboratório de 96

Geotecnia e Meio Ambiente do DEC da PUC-Rio.

Figura 56: Preparação das amostras para o ensaio de cisalhamento direto. 97

Figura 57: Preparação das amostras. 98

Figura 58: Equipamento utilizado nos ensaios de compressão simples. 99

Figura 59: Corpo de prova antes e após o ensaio de compressão simples. 99

$\begin{array}{ll}\text { Figura 60: Equipamento utilizado. } & 100\end{array}$

Figura 61: Saturação das linhas de fluxo. 101

$\begin{array}{ll}\text { Figura 62: Teste da membrana. } & 101\end{array}$

Figura 63: Montagem e acomodação da amostra. 102

$\begin{array}{ll}\text { Figura 64: Colocação da membrana. } & 102\end{array}$

$\begin{array}{ll}\text { Figura 65: Enchimento da Câmara } & 103\end{array}$

Figura 66: Equipamento do Ensaio de Contração. 105

$\begin{array}{ll}\text { Figura 67: Curva granulométrica do solo em estudo. } & 108\end{array}$

Figura 68: Carta de Plasticidade de Casagrande. 109

Figura 69: Análise qualitativa, espectro experimental do solo. 111

Figura 70: Difratograma da amostra de solo natural. 113

Figura 71: Difratograma da amostra de solo passante na peneira \#200. 114

Figura 72: Difratograma da amostra de solo passante na peneira \#400. 114

Figura 73: Difratogramas reunidos, amostra total, material passante nas 115 peneiras \#200 e \#400.

Figura 74: Curvas de compactação com diferentes energias, com reuso de 116 material.

Figura 75: Curvas de compactação com diferentes energias, sem reuso de material.

Figura 76: Curvas de compactação com e sem reuso.

Figura 77: Variação da condutividade hidráulica do solo compactado na 120 Energia do Proctor Normal.

Figura 78: Resultado da análise de MEV com ampliação de 100 (cem) vezes para o ponto $\mathrm{P} 01$. 
Figura 79: Resultado da análise de MEV com ampliação de 100 (cem) vezes para o ponto $\mathrm{P} 05$.

Figura 80: Resultado da análise de MEV com ampliação de 4000 (quatro mil) vezes para o ponto P01.

Figura 81: Resultado da análise de MEV com ampliação de 4000 (quatro mil) vezes para o ponto P05.

Figura 82: Condutividade hidráulica do solo compactado na Energia do Proctor Intermediária.

Figura 83: Condutividade hidráulica do solo compactado na Energia do Proctor Modificada.

Figura 84: Resultado da análise de MEV com ampliação de 100 (cem) vezes para o ponto P01, Energia do Proctor Modificada.

Figura 85: Resultado da análise de MEV com ampliação de 100 (cem) vezes para o ponto P05, Energia do Proctor Modificada.

Figura 86: Resultado da análise de MEV com ampliação de 4000 (quatro mil) vezes para o ponto P01, Energia do Proctor Modificada.

Figura 87: Resultado da análise de MEV com ampliação de 4000 (quatro mil) vezes para o ponto P05, Energia do Proctor Modificada.

Figura 88: Condutividade hidráulica do solo nas energias do Proctor normal, intermediária e modificada em função do teor de umidade de moldagem.

Figura 89: Condutividade hidráulica do solo nas energias do Proctor normal, intermediária e modificada em relação ao teor de umidade ótima.

Figura 90: Variação da condutividade hidráulica devido à variação da energia de compactação.

Figura 91: Resultado da análise de MEV com ampliação de 100 (cem) vezes para o ponto A.

Figura 92: Resultado da análise de MEV com ampliação de 100 (cem) vezes para o ponto $\mathrm{B}$.

Figura 93: Resultado da análise de MEV com ampliação de 4000 (quatro mil) vezes para o ponto $A$.

Figura 94: Resultado da análise de MEV com ampliação de 4000 (quatro mil) vezes para o ponto $\mathrm{B}$. 
Figura 95: Resultado da análise de MEV com ampliação de 100 (cem) vezes para o ponto $\mathrm{C}$.

Figura 96: Resultado da análise de MEV com ampliação de 100 (cem) vezes para o ponto $\mathrm{D}$.

Figura 97: Resultado da análise de MEV com ampliação de 4000 (quatro mil) vezes para o ponto $\mathrm{C}$.

Figura 98: Resultado da análise de MEV com ampliação de 4000 (quatro mil) vezes para o ponto $\mathrm{D}$.

Figura 99: Relação entre a condutividade hidráulica e o índice de vazios.

Figura 100: Relação entre a condutividade hidráulica e o índice de vazios. 136

Figura 101: Relação entre a condutividade hidráulica e o índice de vazios. 137

Figura 102: Valores de Contração com o tempo, para Energia do Proctor 138 Normal.

Figura 103: Valores de Contração com o tempo, para Energia do Proctor Intermediária.

Figura 104: Valores de Contração com o tempo, para Energia do Proctor Modificada.

Figura 105: Relação entre as curvas de compactação e de contração axial, para a Energia do Proctor Normal.

Figura 106: Relação entre as curvas de compactação e de contração axial, para a Energia do Proctor Intermediária.

Figura 107: Relação entre as curvas de compactação e de contração axial, para a Energia Modificada.

Figura 108: Valores de contração axial máxima para os corpos de prova em função do teor de umidade.

Figura 109: Resultados dos ensaios de contração axial em função da umidade ótima.

Figura 110: Resultados dos ensaios de contração diametral.

Figura 111: Resultados dos ensaios de contração volumétrica em relação ao teor de umidade ótima.

Figura 112: Curvas do ensaio de compressão simples para Energia do Proctor Normal.

Figura 113: Curvas do ensaio de compressão simples para Energia do 
Proctor Intermediária.

Figura 114: Curvas do ensaio de compressão simples para Energia do

Proctor Modificada.

Figura 115: Compressão Simples em função do teor de umidade ótima.

Figura 116: Compressão Simples em função do teor de umidade.

Figura 117: Envoltórias de resistência para os pontos da Energia do Proctor Normal.

Figura 118: Ângulo de resistência ao cisalhamento em relação à umidade ótima para Energia do Proctor Normal.

Figura 119: Coesão em relação à umidade ótima para a Energia do Proctor Normal.

Figura 120: Envoltórias de resistência para os pontos compactados na Energia do Proctor Intermediária.

Figura 121: Ângulo de resistência ao cisalhamento em relação à umidade ótima para Energia do Proctor Intermediária.

Figura 122: Coesão em relação à umidade ótima para Energia do Proctor Intermediária.

Figura 123: Envoltórias de resistência para os pontos moldados na Energia do Proctor Modificada.

Figura 124: Ângulo de resistência ao cisalhamento em relação à umidade ótima para Energia do Proctor Modificada.

Figura 125: Coesão em relação à umidade ótima para Energia do Proctor Modificada.

Figura 126: Relação entre a coesão e o teor de umidade, nas três energias de compactação.

Figura 127: Relação entre a coesão e o teor de umidade ótimo, nas três energias de compactação.

Figura 128: Relação entre o ângulo de resistência ao cisalhamento e o teor de umidade, nas três energias de compactação.

Figura 129: Relação entre o ângulo de resistência ao cisalhamento e o teor de umidade ótimo, nas três energias de compactação.

Figura 130: Área aceitável considerando a condutividade hidráulica, onde os símbolos abertos representam valores de $\mathrm{k} \leq 10-9 \mathrm{~m} / \mathrm{s}$ e símbolos 
fechados representam $\mathrm{k} \geq 10-9 \mathrm{~m} / \mathrm{s}$.

Figura 131: Área aceitável considerando a resistência, onde os símbolos

abertos representam valores acima de $200 \mathrm{KPa}$ símbolos fechados representam valores abaixo de $200 \mathrm{KPa}$.

Figura 132: Área aceitável considerando os parâmetros de condutividade 162 hidráulica e de resistência.

Figura 133: Resultados das análises de MEV do Ponto 01 da Energia do

Proctor Normal com ampliação de, 100 e 1000 vezes.

Figura 134: Resultados das análises de MEV do Ponto 01 da Energia do

Proctor Normal com ampliação de 2000 e 4000 vezes.

Figura 135: Resultados das análises de MEV do Ponto 05 da Energia do

Proctor Normal com ampliação de, 100 e 1000 vezes.

Figura 136: Resultados das análises de MEV do Ponto 05 da Energia do

Proctor Normal com ampliação de 2000 e 4000 vezes.

Figura 137: Resultados das análises de MEV do Ponto 02 da Energia do 175

Proctor Intermediária com ampliação de, 100 e 1000 vezes.

Figura 138: Resultados das análises de MEV do Ponto 02 da Energia do

Proctor Intermediária com ampliação de, 2000 e 4000 vezes.

Figura 139: Resultados das análises de MEV do Ponto 01 da Energia do

Proctor Modificada com ampliação de, 100 e 1000 vezes.

Figura 140: Resultados das análises de MEV do Ponto 01 da Energia do

Proctor Modificada com ampliação de, 2000 e 4000 vezes.

Figura 141: Resultados das análises de MEV do Ponto 05 da Energia do

Proctor Modificada com ampliação de, 100 e 1000 vezes.

Figura 142: Resultados das análises de MEV do Ponto 05 da Energia do

Proctor Modificada com ampliação de, 2000 e 4000 vezes.

Figura 143: Relação entre a contração, temperatura e umidade relativa do ar para o ponto 01 da Energia do Proctor Normal.

Figura 144: Relação entre a contração, temperatura e umidade relativa do ar para o ponto 02 da Energia do Proctor Normal.

Figura 145: Relação entre a contração, temperatura e umidade relativa do ar para o ponto 03 da Energia do Proctor Normal.

Figura 146: Relação entre a contração, temperatura e umidade relativa do 
ar para o ponto 04 da Energia do Proctor Normal.

Figura 147: Relação entre a contração, temperatura e umidade relativa do ar para o ponto 05 da Energia do Proctor Normal.

Figura 148: Relação entre a contração, temperatura e umidade relativa do ar para o ponto 01 da Energia do Proctor Intermediária.

Figura 149: Relação entre a contração, temperatura e umidade relativa do ar para o ponto 02 da Energia do Proctor Intermediária.

Figura 150: Relação entre a contração, temperatura e umidade relativa do ar para o ponto 03 da Energia do Proctor Intermediária.

Figura 151: Relação entre a contração, temperatura e umidade relativa do ar para o ponto 04 da Energia do Proctor Intermediária.

Figura 152: Relação entre a contração, temperatura e umidade relativa do ar para o ponto 05 da Energia do Proctor Intermediária.

Figura 153: Relação entre a contração, temperatura e umidade relativa do ar para o ponto 01 da Energia do Proctor Modificada.

Figura 154: Relação entre a contração, temperatura e umidade relativa do ar para o ponto 02 da Energia do Proctor Modificada.

Figura 155: Relação entre a contração, temperatura e umidade relativa do ar para o ponto 03 da Energia do Proctor Modificada.

Figura 156: Relação entre a contração, temperatura e umidade relativa do ar para o ponto 04 da Energia do Proctor Modificada.

Figura 157: Relação entre a contração, temperatura e umidade relativa do 188 ar para o ponto 05 da Energia do Proctor Modificada.

Figura 158: Curvas de Tensão x Deslocamento Horizontal para o ponto 01 da Energia do Proctor Normal.

Figura 159: Curvas de Tensão x Deslocamento Horizontal para o ponto 02 da Energia do Proctor Normal.

Figura 160: Curvas de Tensão x Deslocamento Horizontal para o ponto 03 da Energia do Proctor Normal.

Figura 161: Curvas de Tensão x Deslocamento Horizontal para o ponto 04 da Energia do Proctor Normal.

Figura 162: Curvas de Tensão x Deslocamento Horizontal para o ponto 01 da Energia do Proctor Intermediária. 
Figura 163: Curvas de Tensão x Deslocamento Horizontal para o ponto 02 da Energia do Proctor Intermediária.

Figura 164: Curvas de Tensão x Deslocamento Horizontal para o ponto 03 da Energia do Proctor Intermediária.

Figura 165: Curvas de Tensão x Deslocamento Horizontal para o ponto 04 da Energia do Proctor Intermediária.

Figura 166: Curvas de Tensão x Deslocamento Horizontal para o ponto 05 da Energia do Proctor Intermediária.

Figura 167 Curvas de Tensão x Deslocamento Horizontal para o ponto 01 da Energia do Proctor Modificada.

Figura 168: Curvas de Tensão x Deslocamento Horizontal para o ponto 02 da Energia do Proctor Modificada.

Figura 169: Envoltória de resistência para o ponto 03 da Energia do Proctor Modificada.

Figura 170: Curvas de Tensão x Deslocamento Horizontal para o ponto 04 da Energia do Proctor Modificada.

Figura 171: Curvas de Tensão x Deslocamento Horizontal para o ponto 05 da Energia do Proctor Modificada. 


\section{Lista de Abreviaturas}

ABLP - Associação Brasileira de Resíduos Sólidos e Limpeza Pública

ABNT - Associação Brasileira de Normas Técnicas

ABRELP - Associação Brasileira de Empresas de Limpeza Pública e Resíduos

Especiais

CETESB - Companhia Ambiental do Estado de São Paulo

CONAMA - Conselho Nacional do Meio Ambiente

CPRM - Serviço Geológico do Brasil

DNPM - Departamento Nacional de Produção Mineral

EMBRAPA - Empresa Brasileira de Pesquisa Agropecuária

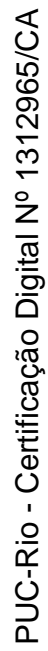

FEAM - Fundação Estadual do Meio Ambiente do Estado de Minas Gerais

IBGE - Instituto Brasileiro de Geografia e Estatística

INMET - Instituto Nacional de Meteorologia

INPA - Instituto Nacional de Pesquisas da Amazônia

IPT - Instituto de Pesquisas Tecnológicas do Estado de São Paulo

ISSMGE - International Society for Soil Mechanics and Geotechnical

Engineering

LCPC - Laboratoire Central des Ponts et Chaussées

MMA - Ministério do Meio Ambiente

MPRR - Ministério Público do Estado de Roraima

SEDU - Secretaria de Estado do Desenvolvimento Urbano

SNIS - Sistema Nacional de Informações sobre Saneamento

SUCS - Sistema Unificado de Classificação de Solos 